\title{
BLADDER NECK OBSTRUCTION IN GENERAL PRACTICE
}

\author{
By GEORGE Y. FEGGETTER, M.S., F.R.C.S.
}

(Surgical First Assistant, British Post-Graduate Medical School).

During the past few years there has been an increase in the number of $\mathbb{\Phi}$ patients suffering from prostatic disorders, and, as the average duration of life $₫$ lengthens, a still further increase is to be expected unless, of course, it becomes possible to control the conditions by the internal administration of hormones. It is the purpose of this article to describe briefly the commoner chronic disorders of the prostate causing urinary obstruction, and to discuss their symptoms and treatment.

The normal prostate is a musculoglandular structure whose functions are unknown. It is believed that its secretions activate the spermatoza. It is about the size and shape of a horse chestnut and lies like an inverted dome below the neck of the bladder. It is traversed by the first one and a quarter inches of urethra; behind it is the rectum and this allows digital palpation of its posterior surface; below and in front is the obliquely placed symphysis pubis and triangular ligament. Above the level of the prostate and behind the bladder neck are the seminal vesicles, and the two ejaculatory ducts pierce the prostatic substance to enter the floor of the urethra near the prostatic utricle at the junction of the middle and distal thirds. The prostate is best described as five portions fused together. These parts or lobes lie, one in front of the urethra, one on each side of the urethra (right and left lobes), one above and between the ejaculatory ducts (the middle lobe) and one, the posterior lobe, behind and below the ejaculatory ducts.

VARIETIES OF PROSTATIC DISEASE.

There are three main types of chronic prostatic disease.

I. Enlargement of one or more lobes.

a. General enlargement of all the lobes of the prostate.

b. Middle lobe enlargement alone.

c. Lateral lobe enlargement alone.

d. Combinations of above.

2. Fibrosis of musculature of bladder neck (sclerotic bladder neck). Median bar, or fibrous prostate.

3. Malignant disease of prostate.

These all give rise to certain symptoms which are characteristic and are best described collectively as "Prostatism." Microscopic pathological details are of little value to the general practitioner, but for his correct interpretation of symptoms and signs, it is essential for him to understand the following macroscopic groups. 
I. (a) A general enlargement of the prostate bulges back into the rectum. It is bigger than normal from side to side and from above downwards. It may be impossible to reach the upper border with the finger in the rectum. As it enlarges upwards it lifts up the bladder base. The prostatic urethra is elongated and kinked. A generalized enlargement of the prostate may be entirely beneath the bladder, or may be associated with one of the following forms and be partially subvesical and partially intravesical.

(b) Middle lobe enlargement of the prostate projects up into the bladder, distorting and hampering the action of the internal sphincter. (There are two sphincters of the lower urinary tract, one, the internal, derived from circular fibres of the bladder at its neck, is involuntary muscle and controlled by the autonomic nervous system. The other, the external sphincter, surrounding the urethra as it passes through the triangular ligament, a voluntary muscle under control of the central nervous system.) This middle lobe may project forwards like a small anteflexed and anteverted uterus, and overlie the internal urethral orifice like a flap valve.

(c) The lateral and middle lobes may enlarge simultaneously and project upwards into the bladder as the cervix uteri projects into the vagina, forming a collar type of enlargement. One lateral lobe may enlarge more than the other, thus kinking the urethra sideways as well as in an antero-posterior direction.

2. Fibrosis of the internal sphincter or adjacent submucous glands causes the condition known commonly as sclerotic bladder neck. Median bar is a condition in which the fibrosis is posterior, and a ridge forms across the posterior part of the internal urethral orifice. These conditions are associated with a small shrunken prostate.

3. Malignant disease of the prostate usually involves the posterior lobe (a part of the prostate which is rarely the site of pathological benign enlargement), but it may begin in any part.

These conditions of the prostate obstruct the outflow of urine during micturition. The bladder muscle hypertrophies. It is thicker, the trabeculations become pronounced and between the interlacing bundles pouching of the mucosa occurs. If a diverticulum is present, it increases in size. After a time the hypertrophy is insufficient to cope with the increasing degree of obstruction, and dilatation of the bladder results. The bladder is unable to empty itself completely, and there is a permanent pool of residual urine.

As the result of interference with the outflow of urine from the bladder the renal function is impaired, probably at first from reflex nervous causes. Later, there is dilatation of the ureters, and a bilateral hydronephrosis.

Stasis of bodily secretions predisposes to infection, and infection of the urinary tract is a serious complication of obstruction.

Calculi may form and grow in bladders with residual urine. 


\section{SYMPTOMS.}

A patient suffering from benign prostate obstruction complains to his medical attendant of one or more of the following group of symptoms:

I. Increased frequency of micturition.

2. Difficulty in the act of micturition.

3. Acute retention of urine.

4. Hæmaturia.

5. Pain.

6. Symptoms of pyelonephritis and cystitis.

7. Manifestations of uræmia.

8. Symptoms of bladder diverticulum.

9. Symptoms of vesical calculus.

Io. Sexual symptoms.

II. Hæmorrhoids and hernia.

Increased Frequency of Micturition. This is often an early symptom due possibly to the increasing size of the prostate raising the trigone and acting as a constant stimulus to micturition, or it may be due to mechanical interference with the internal sphincter allowing urine to be constantly present in a congested posterior urethra. Later in the course of the disease, it is due to chronic urinary retention causing diminished bladder capacity, or is due to inflammation of the bladder causing increased irritability. Actual polyuria due to renal damage causes further increased frequency in late cases.

The frequency is noticed chiefly at night time, the patient having to micturate once or more during the eight hours of rest. In advanced cases the patient's sleep is interrupted hourly or even half hourly.

Difficulty of Micturition. There is some difficulty in starting the stream, causing delay: sometimes, especially in sclerotic bladder necks, the patient must strain to initiate the stream, more commonly he achieves his object in an easier manner by relaxation.

There may be interruption of the stream. This is suggestive of a calculus complicating the disorder, but may also occur when a middle lobe enlargement projects forwards over the internal orifice.

The stream itself, due to urethral kinking and poor bladder tone, may lose its projectile force, and is described as dribbling.

The patient may state that there is no lack of propulsive force, and it is only on inspection of the stream that his error is discovered: often these patients ejaculate the urine by using the perineal muscles. Some patients find that after apparently emptying the bladder, they have a recurrence of the desire to micturate after only a short interval, and pass a quantity of urine equal to the first. This is suggestive of a diverticulum of the bladder complicating the prostatic obstruction.

Pain. Pain is not a prominent symptom of the disease: indeed, many patients progress to uræmic coma without suffering any pain, but there are patients who complain of burning sensations along the urethra, aching pains in the abdomen, 
and aching in the loins. Constant pain in the lower part of the sacrum and over the coccyx is suggestive of prostatic disease, especially the malignant variety. Occasionally a patient is seen whose only symptom is a constant ache in the heels. Other pains occur in the presence of complications, calculus, infection, etc.

Leakage of Urine. Some patients have chronic retention of urine with overflow before they seek medical advice. They have greatly increased frequency and precipitate micturition. There is often leakage of urine at night. To the question "Do you have any difficulty in passing water?" they reply: "No, doctor, the water comes away freely," or "No, the water is too free."

Hæmaturia. This is a rare symptom. Bleeding is from the veins over the vesical surface of the prostate. Sometimes it is so severe that the bladder becomes distended with blood clot which the patient cannot spontaneously void.

Acute Retention of Urine. This often occurs after strenuous exercise, horse riding, cycling, or after excessive eating or drinking, especially where the call for micturition has been neglected. It may follow sexual excesses, but it is seen more often when there has been prolonged sexual stimulation without natural relief. Usually the victim of the retention is one who has had prostatism for some months, but occasionally acute retention occurs in a patient who has previously had no symptoms of prostatic disease, and his prostate is found to be of the generally enlarged subvesical type.

Acute Cystitis and Pyelonephritis. Infection of the urinary tract may be blood-spread to the kidney from a distant focus of infection, but is especially liable to follow careless instrumentation. When infection supervenes the general condition of the patient deteriorates rapidly. He has a parched furred tongue, dry yellowish coloured skin, is feverish and has occasional rigors. He has thirst, but at the same time a peculiar distaste for fluids with which to quench it. There is greatly increased frequency of micturition. He has disturbed nights from halfhourly urgent calls to pass small and unsatisfactory quantities of urine, and soon becomes a haggard, hopeless wreck whose greatest wish is for an early release by death from his torment.

Manifestations of Uræmia. Mental deterioration is one of the earliest symptoms to be noticed: inability to concentrate and to solve simple problems, a feeling of being incapable of proper judgment, a serious handicap in a business man. Then the patient begins to get constant headaches, especially in the morning, he loses weight, has a dry skin and tongue. Uræmic patients sometimes develop symptoms like those of chronic intestinal obstruction, increasing constipation, abdominal distension, loss of appetite and loss of weight, and occasional diarrhœa.

It is not rare to see patients with uræmia due to prostatic enlargement who have had a preliminary investigation of their intestinal tract to exclude carcinoma of the colon, and occasionally a patient is seen who has had an abdominal exploration for the same condition.

Sexual Symptoms. With chronic congestion of the prostate there may be increased libido. This increased sexual desire causes a continual mental conflict, and at times is intolerable, leading respectable elderly men to commit street and other offences. Other patients have diminished libido, and are impotent. 


\section{ROUTINE EXAMINATION.}

When a patient has any of the foregoing symptoms, a thorough examination should be made, conducted in a systematic manner, so that nothing is overlooked, and the results noted. A full history of the complaint should be taken, particulars of the symptoms and their duration, of previous diseases especially venereal ones. The patient should then be asked to pass his urine in the sight of the attendant, and the type of stream noted. This specimen should be collected and tested for albumin, pus, organisms and sugar.

The pupils and knee jerks are examined to exclude gross nervous diseases giving rise to symptoms resembling prostatic obstruction, tabes, G.P.I. disseminated sclerosis, etc.

General examination is made with special reference to the skin, tongue, heart and blood pressure.

Abdominal Examination. Palpate loins for renal enlargement. Palpate suprapubically-partially distended bladder. Examine inguinal regions for hernia. Examine course of urethra to perineum.-A stricture can often be palpated. Examine scrotal contents for evidence of old disease.

Catheterization. A catheter, either rubber or bicoude gum elastic catheter, should be passed, and the amount of residual urine noted. If the bladder is palpable suprapubically or if the patient is frail, this step should be postponed until the patient is at home. Rightly regarded as a minor procedure, catheterization in these cases is nevertheless fraught with great risks. It may precipitate an attack of acute retention or may add an infective element to an aseptic case. Where gross renal disease is present, suppression of urine may result. To minimise these dangers the patient should be in a warm bed and should remain there for the day. He should be given copious draughts of tepid fluid-at least five pints in the first twelve hours following catheterization: It is customary to give tab. hexamine gr. x.t.d.s., and mist. acid sodium phosphate gr. xv.t.d.s., for the following week to keep the urine sterile. It is easier to prevent infection by careful aseptic technique than to cure it by medicaments.

Rectal Examination. With the patient in the knee-elbow position, a systematic examination of the rectum and posterior surface of the prostate is made. The tone of the sphincters or the presence of fibrous piles is noted. The finger palpates the posterior surface of the prostate: the consistence is estimated, is it soft, elastic or hard: is the surface smooth or nodular? The median groove is examined, and the lateral borders felt. An attempt is made to feel the upper border, which cannot be reached in very big prostates. Fixity of the rectal mucosa may possibly occur in very late cases of cancer of the prostate. If the prostate is small, the seminal vesicles are palpable. When all this information has been collected, it is advisable to sweep the finger around the lumen of the rectum, to exclude such conditions as cancer of the bowel itself.

This concludes all that a practitioner can conveniently do in his surgery, though if he is familiar with the use of the cystoscope more facts, useful in treatment, can be obtained. 
The practitioner should then chart his findings on a single sheet as under:-

Name.

Chief Symptoms.

Age.

Duration.

Previous Diseases.

\section{General Examination :}

Weight.

Skin.

Tongue.

Heart.

Blood Pressure.

Pupils.

Knee Jerks.
Gaining, losing or stationary.

Moist or dry.

Moist, dry, furred.

Latent uræmia may be manifested by thirst, dry skin and tongue. Headache. Loss of weight.

To exclude gross nervous diseases as tabes.

\section{Genito-Urinary Examination :}

Frequency.

Reaction to light.

Pain, associated with micturt.

Day / Night.
th micturition.

Before

$\left.\begin{array}{l}\text { During } \\ \text { After }\end{array}\right\}$ the act.

\section{Nature.}

Situation.

Stream.

Good stream or poor.

Difficulty in starting.

Interruption.

Force.

Dribbling.

Urine.

$$
\text { Examine }\left\{\begin{array}{l}
\text { Aibumin. } \\
\text { Sugar. } \\
\text { Deposits. } \\
\text { Pus. }
\end{array}\right.
$$

Specific gravity.

Reaction.

Abdomen. Kidneys. Palpable. Tender.

\section{Bladder.}

Testes. Epididymis and Vas.

Inguinal regions. ?Hernia.

Urethra and Penis.

Prostate. Consistency.

Size.

Borders.

Median groove. Nodules.

Residual Urine. patient.

He can then decide in which of the following groups he should class his

From examination of the rectum:

I. General enlargement.

2. Malignant disease.

3. No enlargement. 
If there is no evident enlargement on rectal examination and the patient has symptoms of prostatism, then he has either a middle lobe or intravesical enlargement, or a condition of sclerotic bladder neck or of median bar formation. This point is stressed because patients are still seen suffering from curable prostatic obstruction, who have been told by their family doctor that they have no prostatic enlargement, as determined by rectal examination, and their symptoms have been ascribed to age, to neurosis, etc., until complications develop which necessitate a review of the case, and the real cause is discovered.

\section{PROGNOSIS.}

A difficult subject in any disease, this is especially so in prostatic disorders. Some patients, without much obstruction, live for many years without the condition progressing; even retrogression may occur though very rarely. Other patients have occasional attacks of acute retention relieved by catheterization, but suffer no ill effects between the attacks. As long as the urine is sterile, and the kidneys are undamaged, the chief complaint is the inconvenience of the increased frequency of micturition. Unfortunately, there is a tendency for the condition to increase in severity; the obstruction becomes greater, nocturnal frequency becomes more marked and interferes seriously with sleep. Diurnal frequency causes the disease to remain constantly in the patient's thoughts. In a fastidious man the continual call to micturition, the occasional precipitate act, the dribbling and even at times leakage of urine, have a very depressing mental effect. He becomes morose, he is dubbed "eccentric", he shuns his former acquaintances, and is unable to follow his earlier pursuits. There is increasing, though insidious, renal damage. Permanent partial retention of urine develops. There is the ever present danger of possible infection. Uræmia or pyelonephritis supervenes, and death results.

\section{TREATMENT.}

The methods of treatment that the practitioner has at his disposal are:-
I. Surgical.
2. Non-surgical.

\section{Surgical.}

In this country the two chief types of operation are:-

(a) Suprapubic prostatectomy.

(b) Transurethral resection of the prostate.

They both attempt to remove the obstruction in such a way as to allow the bladder to be completely emptied, thus preventing further renal damage, preventing or curing infection, and alleviating symptoms. Suprapubic prostatectomy is an attempt to remove the whole adenomatous mass; transurethral resection is the removal of the obstruction only.

Suprapubic Prostatectomy. This operation is well known. It is the method of choice in large prostates when the general condition of the patient is good. Refinements have been recently introduced, such as repair of the prostatic bed with or without complete closure of the bladder, and in consequence of greater pre-operative preparation and care the mortality in expert hands should be not more than five per cent.

Transurethral Resection. This is not yet so well known to practitioners as the suprapubic method. It consists essentially of the passage of an instrument like a cystourethroscope into the bladder along the urethra. A snare-like loop is then passed over the obstructing portions and activated by diathermy. Successive pieces are then reamed out of the prostate using a cutting current, until all 
the obstruction is removed. Bleeding is arrested with a coagulating current, and the instrument removed and replaced by a catheter which remains in for two to four days.

The patient may be expected to micturate normally in four to five days and can return home in seven to fourteen days.

There is no suprapubic incision: It is the method of choice in many cases, but requires much experience and a very high degree of skill for its successful performance.

It has many advantages over the suprapubic method:

1. It is not so severe a procedure. It can be performed satisfactorily using caudal or low spinal anæsthesia.

2. Convalescence is shorter; two to three weeks instead of two to three months.

3. Time in hospital is less; seven to fourteen days instead of three to six weeks.

4. In gravely ill patients, who would not be regarded as justifiable risks for suprapubic prostatectomy, endoscopic resection can be successfully accomplished in two or more sessions.

The disadvantages are:

I. The special skill required. Only competent urologists should attempt the manœuvre. In untrained hands perforation of the bladder, reactionary hæmorrhage, stricture formation and other unsatisfactory results occur.

2. The possibility of recurrence. This is not great, but if it does occur, a second resection can easily be carried out.

The two methods are not, however, rivals in the same field, but should be complementary to each other. Thus:-

The following table gives the chief points of interest regarding the selection of cases:-

\begin{tabular}{|c|c|c|}
\hline & Suprapubic Removal. & Transurethral Resection. \\
\hline $\begin{array}{l}\text { Large } \\
\text { Prostate. }\end{array}$ & $\begin{array}{l}\text { This is the method of } \\
\text { choice. In one or two } \\
\text { stages depending upon } \\
\text { renal function tests. }\end{array}$ & $\begin{array}{l}\text { Not advisable as a routine but is } \\
\text { useful for those patients who } \\
\text { refuse suprapubic operation. } \\
\text { Should be performed in all cases } \\
\text { who would be otherwise con- } \\
\text { demned to permanent supra- } \\
\text { pubic cystotomy. }\end{array}$ \\
\hline $\begin{array}{l}\text { Fibrous } \\
\text { Prostates. } \\
\text { Sclerotic } \\
\text { Bladder } \\
\text { Neck. }\end{array}$ & $\begin{array}{l}\text { Contraindicated. } \\
\text { Removal very difficult } \\
\text { and unsatisfactory. } \\
\text { Wedge excision not very } \\
\text { satisfactory. }\end{array}$ & $\begin{array}{l}\text { The method of choice. } \\
\text { Can cure. }\end{array}$ \\
\hline Malignant. & Prognosis hopeless. & $\begin{array}{l}\text { May obviate the necessity for } \\
\text { suprapubic cystotomy. }\end{array}$ \\
\hline $\begin{array}{l}\text { Early } \\
\text { Enlargement. }\end{array}$ & $\begin{array}{l}\text { Patient averse to a major } \\
\text { procedure. }\end{array}$ & $\begin{array}{l}\text { Relieves symptoms and prevents } \\
\text { progressive obstruction. }\end{array}$ \\
\hline
\end{tabular}


There are certain questions which most patients ask when a practitioner suggests the possibility of an operation.

r. Is the operation necessary? As shown above, this depends on the extent of the disease. Where there is residual urine of five ounces or more, or where the renal function tests show deterioration of the kidneys, or where there is urinary infection, operation is necessary, if life is not to be shortened by the disease. Otherwise the necessity for operation depends on the symptoms the patient has. If renal function tests are normal, and the urine clear, a formidable operation need only be advised if the discomfort is great. On the other hand, it must be pointed out that the patient is in a better condition to stand operation at this time.

2. Is the operation serious? Yes, the operation is serious. In skilled hands the mortality is two to five per cent. ; in late cases who are bad risks and in unskilled hands anything to twenty-five per cent. Endoscopic resection in chosen cases has a slightly lower mortality rate, two per cent.

3. Will it interfere with sex functions? In suprapubic prostatectomy sexual function may or may not be affected. Usually there is little difference than before the operation. In endoscopic resection, there is no difference.

4. Shall I be sterile? Suprapubic prostatectomy usually sterilizes the patient. Endoscopic resection usually leaves the patient quite normal.

5. How long shall I be off work?

After endoscopic resection four to sixe $\overrightarrow{0}$ weeks; after suprapubic prostatectomy three to four months at least, more often six: months. patient.

6. Shall I be cured? Both operations, performed by experts, cure the

\section{Non-Surgical Treatment.}

The practitioner decides that his patient is not suitable for operative intervention. That is, that the general condition of the patient is good, and the symptoms not sufficiently severe to warrant the risks of an "Operation de complaisence", or that the general condition of the patient is such, owing to extreme age, other disease, etc., that the risks of operation are greater than the risks of the disease.

If he is holding a watching brief, in the case of a patient with small prostatic enlargement whose obstructive symptoms are absent or slight, he should keep an accurate record of blood urea and urea concentration test, and the amount of residual urine, if any. This should be repeated twice a year.

Diet and Hygiene. There is no special diet to be recommended. Most patients eat more than is necessary, and derive much benefit from the prescription of moderate diet. Highly spiced foods, rich meats, sweetbreads, etc., should be prohibited; otherwise there is no advantage in cutting out meat.

Fluids. Water, weak tea, etc., are good in aiding elimination by the kidneys - five to ten pints per day, beers one pint per day, do no harm. Spirits are best avoided. Excess of any kind is liable to result in an attack of acute retention. 
Sexual Habits. Here again, moderation. The patient should keep to his usual habits.

Massage of Prostate. This I think, is of little benefit in these cases.

Drugs. (a) To diminish the size of the prostate. Magnesium and its salts have a good reputation in the matter. Extracts of ductless glands are advertised, but no accurate satisfactory reports on their action are available as yet.

(b) Urinary Antiseptics.

Hexamine and acid sodium phosphate are excellent antiseptics, but it must be remembered that Hexamine itself in certain patients produces a chemical cystitis. Pyridium, neotropin and cystopurin are proprietary preparations which, though expensive, may be useful in the prevention and cure of infection.

Progress. The patient should be çatheterized every six months to record the residual urine.

\section{Treatment of Complications.}

Acute Retention of Urine. This should be relieved as soon as possible. If hot baths have failed, the passage of a rubber catheter is necessary. If this fails, a bicoude gum elastic catheter is used and is always successful. In cases of very big prostates with a distorted urethra, slight rotatory movements of the catheter enable it to be manipulated past the kinks.

Chronic Retention. When the patient has a bladder distended to the umbilicus, complaining of marked frequency or leaking (retention with overflow), and if for any reason operation is contraindicated, he should be decompressed, that is, a gum elastic catheter inserted, and $5 \mathrm{oz}$. of urine drawn off hourly. When the bladder is empty, he should be catheterized daily and the bladder washed out with boric lotion, potassium permanganate $\frac{1}{10,000}$, or oxycyanide of mercury $\frac{1}{10,000}$, or iodine, a drachm to a pint of water.

Acute Infection. An indwelling catheter should be inserted and the bladder washed out every four hours with one of the above lotions.

Suprapubic Cystostomy (Permanent). This should be a thing of the past, but in certain parts of the country there are many patients who end their days in conditions of abject misery owing to neglect of a permanent suprapubic cystostomy.

These should have daily lavage with oxycyanide of mercury. Often the patient lives at such a distance from the doctor as to be beyond his care. It is most important that, if relatives fulfil this function, they should be properly instructed in aseptic methods. Citrates must not be given. Citrates in these cases cause an alkaline encrusted cystitis, and make the symptoms worse. Acid sodium phosphate or ammonium chloride should be prescribed. If an alkaline encrusted cystitis is present, the bladder should be washed out with dilute hydrochloric acid solution. The tube should be removed every two months and thoroughly cleaned mechanically, resterilized and reinserted. 